\title{
A review of ramelteon in the treatment of sleep disorders
}

\author{
David N Neubauer \\ Department of Psychiatry, Johns \\ Hopkins University School of \\ Medicine
}

Correspondence: David N Neubauer Johns Hopkins Bayview Medical Center, 4940 Eastern Avenue, Box 15I, Baltimore, MD 21224, USA

Tel +l 4I05500066

Email neubauer@jhmi.edu

\begin{abstract}
Ramelteon is a selective melatonin receptor $\left(\mathrm{MT}_{1}\right.$ and $\mathrm{MT}_{2}$ ) agonist that has been approved by the US Food and Drug Administration for the treatment of insomnia characterized by difficulty with sleep onset. It is the only approved sleep-promoting medication that does not have a direct sedating effect, but rather enhances sleep through effects on sleep regulatory mechanisms within the suprachiasmatic nucleus. Ramelteon has been shown to have no abuse liability and therefore is not scheduled by the U.S. Drug Enforcement Agency as a controlled substance. It is available as an $8 \mathrm{mg}$ tablet, which should be taken approximately 30 minutes prior to bedtime. The FDA approval contains no limitation on how long the medication may be prescribed.
\end{abstract}

Keywords: circadian rhythm, insomnia, melatonin receptors, ramelteon, sleep-wake cycle

\section{Introduction}

Insomnia, the most common sleep-related complaint, is an occasional problem for approximately one third of the general population. Ten to $15 \%$ of adults suffer with chronic insomnia. The prevalence is higher among elderly individuals and in people with comorbid medical and psychiatric disorders. Chronic insomnia is associated with impaired daytime functioning, decreased quality of life, and increased health care utilization.

Insomnia sufferers seek relief with a remarkable array of substances, including alcohol, herbal and homeopathic preparations, dietary supplements, and over-the-counter antihistamines. Health care providers may recommend an assortment of sedating psychotropic medications as primary treatments for insomnia. Among these are antidepressants (eg, trazodone, doxepin), antipsychotics (eg, quetiapine), anticonvulsants (eg, gabapentin, tiagabine), and higher-dose antihistamines (eg, diphenhydramine). Convincing evidence is lacking for the efficacy in the treatment of chronic insomnia of all of the above compounds (National Institutes of Health 2005). Additionally, there are significant safety concerns associated with each of these approaches.

In June 2005, a US National Institutes of Health (NIH)-sponsored panel reviewed the published evidence regarding the treatment of chronic insomnia (National Institutes of Health 2005). The panel concluded that evidence supports only cognitive behavioral therapy and the US Food and Drug Administration (FDA)-approved benzodiazepine receptor agonist (BZRA) hypnotic medications. At the time of this panel statement, ramelteon had not yet been approved, and little efficacy data were available for review.

For several decades the BZRA hypnotic medications were the only medications approved by the FDA for the treatment of insomnia. Initially, all of the BZRAs structurally were benzodiazepines. In the early 1990s, non-benzodiazepine BZRAs became available and ultimately became the most widely prescribed hypnotics. However, many new pharmacologic agents with novel mechanisms of action have been investigated 
Table I Ramelteon: key characteristics

\begin{tabular}{|c|c|}
\hline Brand name & Rozerem \\
\hline FDA spproval & July 2005 \\
\hline DEA dtatus & Unscheduled \\
\hline Indication & $\begin{array}{l}\text { For the treatment of insomnia } \\
\text { characterizedby difficulty with sleep } \\
\text { onset }\end{array}$ \\
\hline Mechanism of action & Selective melatonin receptor agonist \\
\hline $\begin{array}{l}\text { Peak concentration time } \\
\text { (median) }\end{array}$ & 45 minutes \\
\hline $\begin{array}{l}\text { Elimination half-life } \\
\text { (approximate) }\end{array}$ & $\mathrm{I}-2.6$ hours \\
\hline Available doses & $8 \mathrm{mg}$ \\
\hline Prescribing recommendation & $8 \mathrm{mg} 30$ minutes prior to bedtime \\
\hline \multirow[t]{2}{*}{ Warnings } & Moderate to severe hepatic impairment \\
\hline & Coadministration with fluvoxamine \\
\hline \multirow[t]{3}{*}{ Adverse events } & Somnolence $(5 \%)$ \\
\hline & Dizziness (5\%) \\
\hline & Fatigue $(4 \%)$ \\
\hline
\end{tabular}

as possible sleep-promoting agents. Ramelteon is the first of these new mechanism of action sleep-promoting medications to be approved by the FDA.

\section{Sleep-wake cycle regulation}

Normally the timing of sleep and wakefulness are strongly influenced by the combined effects of homeostatic and circadian processes (Richardson 2005). In humans, the homeostatic system promotes sleep for about one third of the time, or a total of approximately 8 hours daily. Sleepiness builds up during waking and is dissipated during sleep. Insufficient sleep leads to increased sleepiness. Following nighttime sleep, there is a steady buildup of sleepiness from the homeostatic process throughout the day and evening until one falls asleep at nighttime again. People generally do not subjectively experience the increasing homeostatic sleep pressure due to the opposing alerting signal from the circadian system, which is promoting maximum arousal during the evening. The late afternoon and evening circadian arousal, coordinated by activity in the suprachiasmatic nuclei, provides the ability for people to remain awake and active for approximately 16 hours. While the homeostatic system promotes the amount of sleep people need, the circadian system optimizes when that sleep best can be achieved. The quality and duration of both wakefulness and sleep may be impaired when the homeostatic and circadian systems are dissociated, as can occur with jet lag and shift work.

The production and release of melatonin by the pineal gland ultimately is regulated by the suprachiasmatic nucleus (SCN) (Turek and Gillette 2004). Typically melatonin is produced and the level rises in the evening as one's usual bedtime is approaching. The melatonin level plateaus during the night and declines later during the night as the end of the normal sleep period approaches. There are two melatonin receptor subtypes in the SCN. Agonists of the $\mathrm{MT}_{1}$ receptor subtype decrease the evening alerting signal and therefore enhance sleep onset. The $\mathrm{MT}_{2}$ receptor subtype serves to reinforce or shift the timing of the circadian system. Under normal circumstances, the dual agonist activity promotes sleep onset directly and also reinforces the regular timing of evening sleepiness and sleep onset.

\section{Ramelteon history}

In 1992 Takeda initiated a plan to research alternate strategies to address the treatment of insomnia. They focused on creating a melatonin receptor agonist. In 1996 TAK-375, now known as ramelteon, was developed. Clinical trials of the compound began in 1999. These trials have included over 7900 unique subjects and over 800 subject-years of use. The studies conducted in the development of ramelteon have included general efficacy and safety protocols, as well as specific studies examining pharmacokinetic and pharmacodynamic characteristics, toxicology, abuse liability, and use in special populations. The New Drug Application for ramelteon was submitted to the FDA in September 2004, and was approved in July 2005. Ramelteon became available for use in the US in September 2005, under the brand name Rozerem.

\section{Chemistry}

Ramelteon ((S)- $\mathrm{N}$-[2-(1,6,7,8-tetrahydro-2H-indeno[5,4-b] furan-8-yl)ethyl]propionamide) is the single (S)-enantiomer of a tricyclic indan derivative. The structure of ramelteon is shown in Figure 1.

\section{Pharmacokinetics}

Ramelteon is rapidly absorbed and reaches a peak serum concentration in less than one hour (Hibberd and Stevenson 2004; Karim et al 2006a). There is extensive first-pass metabolism, and the mean systemic availability following an oral dose is less than $2 \%$ (Amakye et al 2004). In humans, protein binding is approximately $82 \%$ (Takeda Pharmaceuticals North America 2006). Pharmacokinetic studies of ramelteon at a wide range of doses show a large degree of intersubject variability in the peak concentration and total systemic exposure (Karim et al 2006). Administration of ramelteon with food increases the AUC, delays the $\mathrm{T}_{\max }$, and decreases the $\mathrm{C}_{\text {max }}$ (Karim et al 2004a). The onset of action should be faster on an empty stomach; however, this may not 


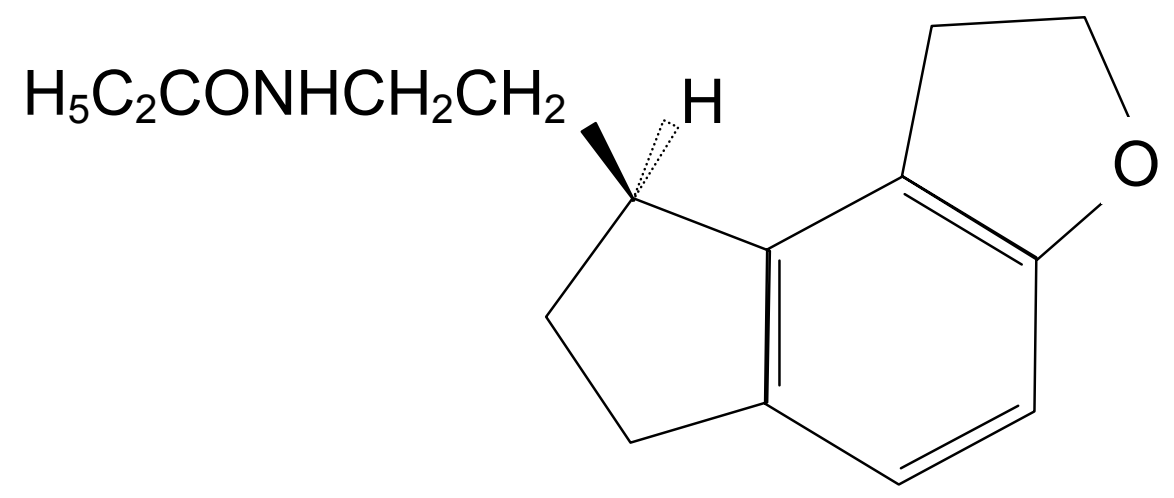

Figure I Structure of ramelteon (Takeda Pharmaceuticals North America 2006).

be clinically significant. The use of ramelteon with or soon after a high-fat meal is not recommended due to the expected delay in absorption.

The metabolism of ramelteon is primarily through the CYP1A2 isozyme and secondarily through the CYP2C subfamily and CYP3A4 isozymes. The elimination half-life for ramelteon generally is under 2 hours in adults and elderly subjects, and is not influenced by gender (Karim et al 2006; Greenblatt et al 2007). Serum measurements in humans identify four metabolites: M-I, M-II, M-III, and M-IV. The only active metabolite is M-II, which has similar pharmacodynamic properties with about one-tenth the potency of ramelteon. The M-II metabolite has the greatest systemic exposure, but has an elimination half-life of approximately 2-4 hours (Karim et al 2006).

\section{Pharmacodynamics}

In vitro receptor binding assays have shown that ramelteon is an agonist of the $\mathrm{MT}_{1}$ and $\mathrm{MT}_{2}$ melatonin receptor subtypes (Kato et al 2005). In comparison with melatonin, ramelteon had significantly greater affinity and selectivity for these binding sites. The dissociation constant $\left(\mathrm{K}_{\mathrm{i}}\right)$ of ramelteon in the in vitro studies was 3-16 times higher than melatonin. In contrast with melatonin, ramelteon had significantly lower affinity for the $\mathrm{MT}_{3}$ binding site, which represents a melatonin-sensitive form of the enzyme quinone reductase 2 that has wide tissue distribution in mammals. In vitro studies also have demonstrated that ramelteon has no measurable affinity for a large number of tested ligand binding sites, including benzodiazepine, dopamine, and opiate receptors.

Preclinical animal studies to assess sleep-related effects have been conducted with freely moving cats (Miyamoto et al 2004) and macaque monkeys (Yukuhiro et al 2004). In the cat study, orally administered ramelteon was compared with exogenous melatonin. Monitoring for sleep characteristics included the electroencephalogram, muscle tension, and eye movements. Ramelteon significantly reduced wakefulness over a wide dosage range with effects lasting up to 6 hours, in contrast to the effects of exogenous melatonin lasting up to 2 hours. Furthermore, ramelteon was found to be about 10 times more potent than melatonin in promoting sleep.

The study with macaques compared the effects of oral doses of ramelteon, exogenous melatonin, and zolpidem (Yukuhiro et al 2004). Ramelteon demonstrated significantly shortened sleep onset latency and increased total duration of sleep, while melatonin had a weaker effect on sleep latency and no effect on sleep duration. No behavior changes were present with ramelteon and melatonin; however, zolpidem at the highest dose was associated with sedation and muscle relaxation.

\section{Efficacy for transient insomnia}

Two studies have investigated the effects of ramelteon compared with placebo in a model of transient insomnia in healthy subjects without sleep complaints. Both have taken advantage of the "first night effect" in a sleep laboratory, when the ability to fall asleep and remain asleep may be challenged in this novel sleep environment. In one clinical trial, 375 subjects were given ramelteon 16 or $64 \mathrm{mg}$, or placebo (Roth et al 2005). The polysomnographic recording demonstrated a significantly shorter latency to persistent sleep and increased total sleep time with both ramelteon doses. The similar sleep latencies found with both doses suggests a flat dose-response curve. The second transient insomnia model study involved 289 healthy subjects who were given ramelteon $8 \mathrm{mg}, 16 \mathrm{mg}$, or placebo (Zammit et al 2005). The polysomnographic recording showed that the subjects taking both doses of the ramelteon fell asleep faster and slept longer to a significant degree in comparison with the placebo group. The latency to persistent sleep for the ramelteon $8 \mathrm{mg}$ and placebo subjects 
were 12.2 and 19.7 minutes, respectively. The total sleep time for the ramelteon $8 \mathrm{mg}$ and placebo groups were 436.8 and 419.7 minutes, respectively.

\section{Efficacy for chronic insomnia}

The efficacy of ramelteon in promoting statistically significant reductions in latency to persistent sleep and increases in total sleep time across a broad dosage range in chronic insomnia subjects was demonstrated in a double-blind, placebocontrolled, five-period crossover study of 107 adult subjects (ages 18-64 years) (Erman et al 2006). Each of the subjects followed a randomized dosing sequence that included two treatment nights with a placebo and ramelteon $4,8,16$, and $32 \mathrm{mg}$. The similar polysomnographically determined sleep latency values over this range of ramelteon dosages suggests a flat dose-response curve. There were no differences among the ramelteon doses and placebo with regard to wake time after sleep onset.

A 5-week, double-blind study of 405 adults (ages 18-64 years) with chronic insomnia incorporated both objective polysomnographic and patient-reported measures (Zammit et al 2007). The subjects were randomized to groups with nightly doses of ramelteon $8 \mathrm{mg}$ or $16 \mathrm{mg}$, or placebo. The 35 treatment nights were preceded by a 1-week single-blind placebo baseline period and were followed by a single-blind 2-night placebo run-out to assess potential withdrawal or rebound (sleep characteristics worse than study baseline values) effects. Sleep laboratory efficacy assessments were performed for 2 nights during weeks 1, 3, and 5. Compared with the placebo group, both ramelteon doses resulted in significantly shorter polysomnographically recorded sleep latency values throughout the treatment period. The patient-reported sleep latencies also were significantly shorter than the placebo at weeks 1, 3, and 5 for the $8 \mathrm{mg}$ dose (Figure 2) and at weeks 1 and 3 for the $16 \mathrm{mg}$ dose. Polysomnographically determined total sleep time and sleep efficiency were statistically greater than placebo for both ramelteon doses at week 1. During subsequent weeks statistical significance with greater total sleep time and sleep efficiency was found only at the $16 \mathrm{mg}$ dose during week 3 . The patient-reported total sleep time was significantly greater for the $8 \mathrm{mg}$ dose during weeks 1,3 , and 5, and for the $16 \mathrm{mg}$ dose during week 1 . There was no evidence of rebound insomnia or withdrawal effects on single-blind placebo run-out discontinuation at either ramelteon dose.

One hundred elderly chronic insomnia subjects (mean age 70.7 years) participated in a three-way crossover study involving ramelteon $4 \mathrm{mg}$ and $8 \mathrm{mg}$ and placebo (Roth et al 2007). Both polysomnographically recorded sleep latency and total sleep time were significantly better in the ramelteon $4 \mathrm{mg}$ and $8 \mathrm{mg}$ groups compared with the placebo group; however, the subjective total sleep time measures did not reach statistical significance. There was a significant reduction in the subjective sleep latency in the $4 \mathrm{mg}$ group.

The efficacy of ramelteon was assessed in 829 older adult (ages 64-93 years) chronic insomnia subjects enrolled in a 5 -week outpatient trial with groups receiving nightly doses of ramelteon $4 \mathrm{mg}, 8 \mathrm{mg}$, or placebo (Roth et al 2006). One-week single-blind placebo periods occurred before and after the double-blind treatment period. Compared with the placebo group, the sleep diary outcomes demonstrated significantly shorter subjective sleep onset latencies for both ramelteon dose groups at week 1 and week 5 , and for the $8 \mathrm{mg}$ group at all three assessments. The $8 \mathrm{mg}$ group was not significantly different from the placebo group for reported total sleep time, although the $4 \mathrm{mg}$ group was significant for the week 1 and 3 assessments. However, the overall treatment effect combining the two ramelteon treatment groups also resulted in significantly better total sleep time relative to the placebo group at weeks 1 and 3. There were no significant differences between ramelteon and placebo regarding sleep quality, number of nighttime awakenings, and ease of falling back to sleep. With discontinuation, there was no evidence of rebound insomnia or withdrawal effects.

A subset including 327 of the above 829 older adults met a criterion for severe sleep initiation difficulty with reported baselines equal to or greater than 60 minutes (Mini et al 2007). In this subanalysis, 157 subjects had been randomized to the ramelteon $8 \mathrm{mg}$ group and 170 to the placebo group. Statistically significant improvement from baseline remained significantly better in the ramelteon group compared with the placebo group at weeks 1 through 5. By week 1 the mean reported sleep latency decreased by 7.5 minutes in the placebo group and 23.2 minutes in the ramelteon group. At week 5 the improvement relative to baseline for the placebo group was 17.1 minutes and for the ramelteon group was 37.4 minutes.

The long-term nightly use of ramelteon was evaluated in a 12-month open-label, non-placebo-controlled outpatient study of 1213 adult and elderly subjects meeting criteria for chronic insomnia (DeMicco et al 2006). The adults (ages 18-64 years) were given ramelteon $16 \mathrm{mg}$, and the elderly subjects (ages 65 and older) were given $8 \mathrm{mg}$. At 6 months, 597 subjects remained in the study, and 473 subjects completed the full 12 months (Richardson et al 2006a). Sleep diary data showed that in comparison with baseline sleep latency estimates, both elderly and adult subjects experienced improvement at month 1 (34.0\% and $35.1 \%$, respectively), 


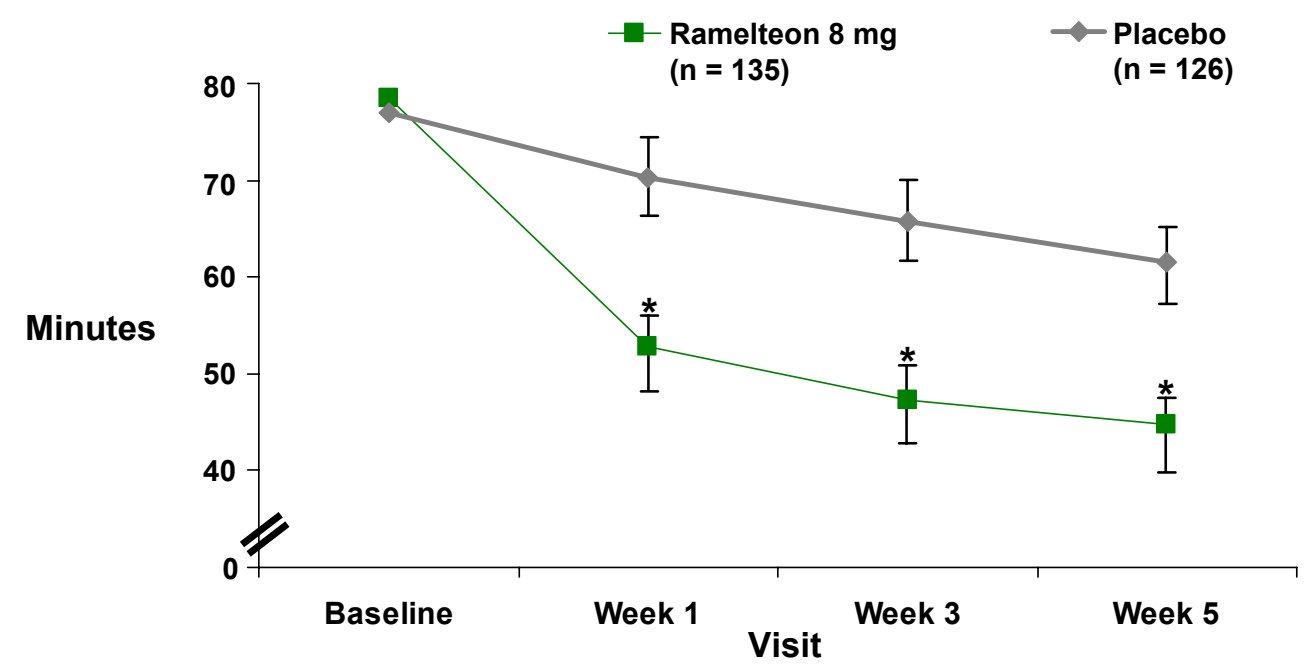

Figure 2 Ramelteon efficacy: Subject-reported sleep latency in adults with chronic insomnia during a 5-week double-blind, placebo-controlled study (derived from data of Zammit et al 2007) ( $\mathrm{p} \leq 0.05)$.

month $6(44.7 \%$ and $49.1 \%)$, and month $12(50.3 \%$ and $52.1 \%$ ) (DeMicco et al 2006). Total sleep time estimates also showed steady improvement for elderly and adult subjects at month 1 (15.2\% and 16.9\%), month 6 (21.6\% and $22.7 \%)$, and month 12 (25.5\% and 23.9\%). Clinical Global Impression assessments also reflected the moderate and sustained improvement. A single-blind 3-day placebo run-out at the end of the study showed no significant prolongation in sleep latency that would reflect rebound insomnia.

The longest assessment of both objective and subjective measures in a placebo-controlled study with the nightly use of a pharmacologic agent in the treatment of chronic insomnia subjects has been reported with a 6-month ramelteon clinical trial (Wang-Weigand et al 2007). A total of 451 adults with chronic insomnia were randomized to ramelteon $8 \mathrm{mg}$ or placebo groups. Two consecutive nights of polysomnographic recording were completed at week 1 and months $1,3,5$, and 6 of treatment, and week 1 of a single-blind placebo runout. Subjective sleep variables were measured with morning questionnaires. Relative to placebo, the ramelteon subjects experienced statistically significantly shorter objective latency to persistent sleep throughout the 6-month study. Subjective sleep latency also was shorter throughout the study and reached statistical significance at week 1 , month 1 , and month 5 . There was no evidence of withdrawal or rebound insomnia on discontinuation.

\section{Efficacy in facilitating circadian phase shifts}

Although human studies examining the efficacy of ramelteon in the treatment of circadian rhythm disorders have not been presented, preliminary investigations suggest possible future applications in this clinical area. In a study with rats, the animals were entrained to a 12-hour light-dark cycle and then subjected to an abrupt 8-hour advance phase shift. The ability of ramelteon or melatonin to facilitate the phase shift in rat activity rhythm was compared with a vehicle control during 14 days of administration at the same time of day (Hirai et al 2005). The primary outcome measure was the number of days required for the animals to exhibit at least $85 \%$ of typical activity during the night for 2 consecutive days. Both ramelteon and melatonin accelerated the reentrainment to the new light-dark cycle following the abrupt phase shift.

The phase-shifting effects in healthy human subjects were examined in a study of 18 subjects during a 4-period crossover study including ramelteon $4 \mathrm{mg}$ and $16 \mathrm{mg}$, melatonin $5 \mathrm{mg}$, and placebo (Richardson et al 2005). The ramelteon doses were effective in producing a phase advance of the endogenous circadian rhythm as indicated by the timing of the evening dim light salivary melatonin onset.

In a subsequent trial, a 5-hour phase advance was imposed on 75 healthy adults who were randomized to receive ramelteon $1 \mathrm{mg}, 2 \mathrm{mg}, 4 \mathrm{mg}, 8 \mathrm{mg}$, or placebo. The subjects were confined in a sleep laboratory under low-intensity light for five nights and six days. Ramelteon $1 \mathrm{mg}, 2 \mathrm{mg}$, and $4 \mathrm{mg}$ produced a significantly greater and more rapid circadian phase advance compared with placebo ( $-80.9 \mathrm{~min}, \mathrm{p}=0.002$; $-73.3 \mathrm{~min}, \mathrm{p}=0.003$; and $83.4 \mathrm{~min}, \mathrm{p}=0.001$, respectively), as indicated by endogenous melatonin secretion offset time; the $8 \mathrm{mg}$ dose showed a phase advance that did not reach statistical significance $(-27.9 \mathrm{~min}, \mathrm{p}=0.392)$ (Richardson et al 2006b). 


\section{Safety and tolerability}

The safety of ramelteon has been examined in numerous preclinical and clinical trials. The efficacy trials have demonstrated a lack of significant next-morning residual sedation and cognitive impairment using questionnaires and measures, such as the digit symbol substitution test and the visual analog scale for alertness. Specific safety studies have focused on potential interactions with ethanol and selected coadministered medications, as well as abuse liability, endocrine effects, and use in special medical populations. There is no evidence of discontinuation withdrawal symptoms. Ramelteon and placebo subjects had similar scores on the Tyrer Benzodiazepine Withdrawal Symptom Questionnaire (Takeda Pharmaceuticals North America 2006). Figure 3 shows the incidence of treatmentemergent adverse events in the clinical studies (Takeda Pharmaceuticals North America 2006). The adverse event items that were reported by at least $2 \%$ more of the ramelteon subjects compared with placebo subjects during the clinical trials included only somnolence (5\% vs 3\%), dizziness ( $5 \%$ vs $3 \%$ ), and fatigue ( $4 \%$ vs $2 \%$ ).

A broad range of safety parameters were monitored during the one-year, open-label, non-placebo-controlled study of ramelteon $16 \mathrm{mg}$ (adults) and $8 \mathrm{mg}$ (older adults) noted above (DeMicco et al 2006; Richardson et al 2006a). No clinically meaningful changes were reported regarding vital signs, physical exams, clinical chemistry, hematology, urinalysis, and electrocardiogram trends. No notable endocrine or sexual/reproductive function changes were observed except for a slight decrease in the free and total testosterone levels in older males on selected monthly visits. The testosterone levels returned to the normal range by the final visit of the study.

The absence of balance difficulty due to ramelteon has been confirmed in three studies. In each report the effects of the medication were no different from placebo, while subjects given active comparators demonstrated evidence of acute impairment in balance. A total of 260 adult chronic insomnia subjects completed a protocol involving 14 nightly doses of placebo, ramelteon $8 \mathrm{mg}$, or zopiclone $7.5 \mathrm{mg}$. On the last night the subjects slept in a laboratory setting and were awakened 1.5-2 hours following the evening dose. The subjects stood on a balance platform and were assessed for sway. The ramelteon subjects were the same as the placebo group; however, the zopiclone group had a significantly worse calculated area of center of pressure on the platform (Hajak et al 2007). Thirty-three older adult chronic insomnia subjects participated in a single-dose crossover study examining the

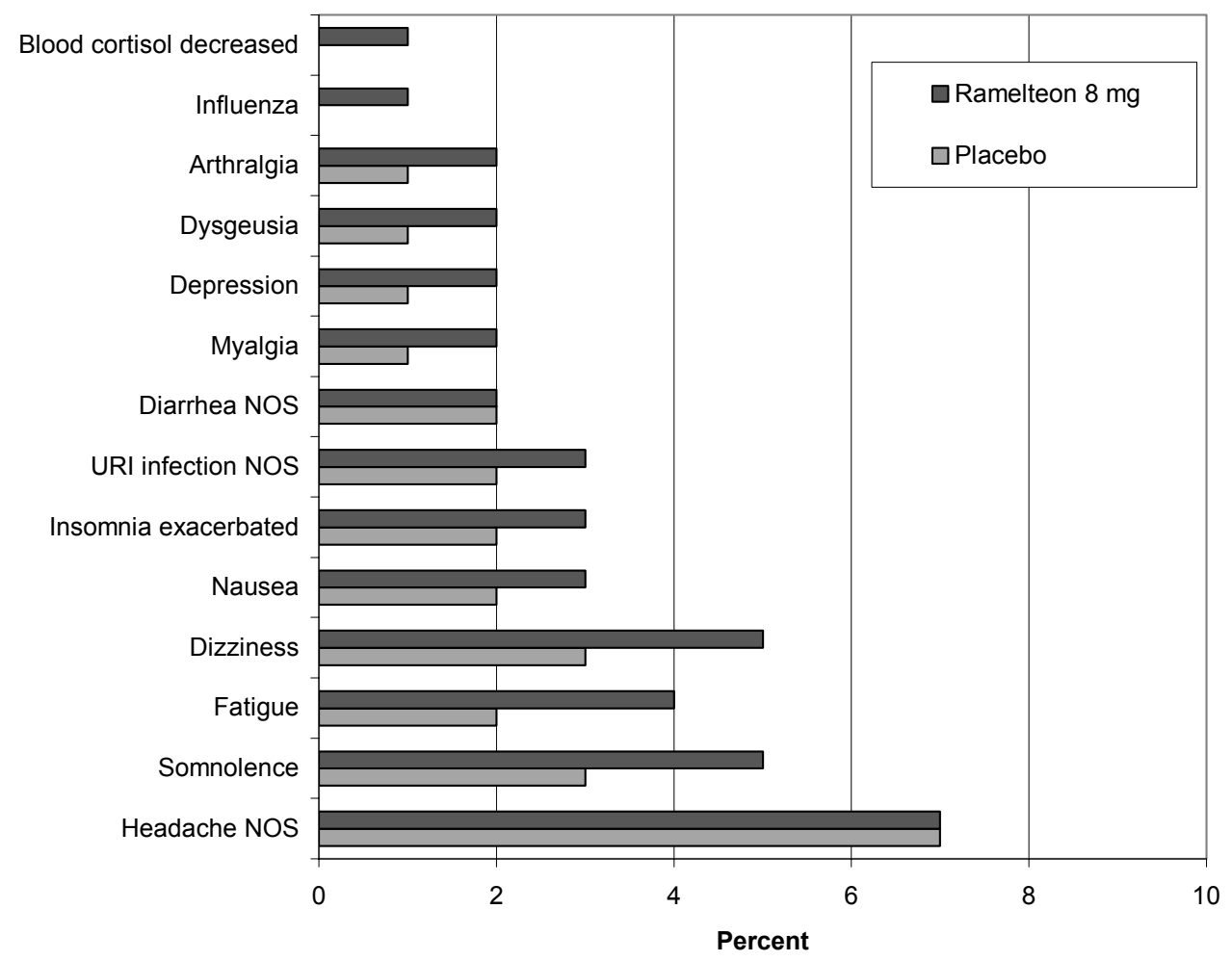

Figure 3 Incidence (\% of subjects) of treatment emergent adverse events in phase I-3 studies (Takeda Pharmaceuticals North America 2006). 
effects of placebo, ramelteon $8 \mathrm{mg}$, and zolpidem $10 \mathrm{mg}$ on balance. Two hours following a bedtime dose, the subjects were awakened and assessed for balance and mobility with computerized dynamic posturography. Only on the zolpidem nights did the subjects exhibit significant impairment relative to the placebo performance (Wang-Weigand et al 2007). Balance was among the outcomes investigated in an abuse liability crossover study of substance abusers given daytime ramelteon (16-160 mg), triazolam $(0.25-0.75 \mathrm{mg})$, and placebo doses. The effects of ramelteon on balance were no different from placebo throughout the wide dose range; however, balance impairment was noted at the higher triazolam doses (Johnson et al 2006).

\section{Pharmacokinetic interaction studies}

Pharmacokinetic studies have examined the possible effects of ramelteon coadministered with commonly prescribed medications or those that are representative of hepatic isozyme inhibitors or inducers. Overall, ramelteon was shown not to have a significant effect on the serum concentration of coadministered medications. In some cases there were statistically significant increases or decreases in the ramelteon level due to the hepatic isozyme inhibition or induction of the coadministered medication. Medications tested that had no significant effect on the systemic exposure of ramelteon included dextromethorphan (Tolbert et al 2004a), digoxin (Tolbert et al 2004b), midazolam (Karim et al 2004b), zolpidem (Karim et al 2007a), and warfarin (Karim et al 2005b). The systemic exposure of ramelteon was decreased by rifampin (Karim et al 2004c) (potent CYP inducer) and sertraline (Karim et al 2006c) and was increased by theophylline (Tolbert et al 2004c) (CYP1A2 substrate), fluoxetine (Sainati et al 2004) (CYP2D6 inhibitor), escitalopram (Karim et al 2006b), donepezil (Karim et al 2007b), omeprazole (Karim et al 2005a), venlafaxine (Karim et al 2006d), fluconazole (Karim et al 2004d) (CYP2C9 inhibitor), and ketoconazole (Karim et al 2004d) (CYP3A4 inhibitor). Coadministration of ramelteon and ethanol resulted in a $50 \%$ increase in the exposure of ramelteon (Karim et al 2004e).

Even with moderate changes in the ramelteon levels from coadministered medications, these interactions generally have not been regarded as clinically significant for the following reasons. Ramelteon undergoes extensive first-pass metabolism, has highly variable plasma concentrations and a wide therapeutic window, and has a flat dose-effect relationship. That it is metabolized by several hepatic isoenzymes also limits the potential for drug interactions. The one medication that may markedly elevate the ramelteon concentration is the potent CYP1A2 isozyme inhibitor, fluvoxamine (Luvox), and therefore combined use is not recommended (Takeda Pharmaceuticals North America 2006).

\section{Special population safety studies}

The safety of ramelteon in patients with mild-to-moderate COPD was examined in a double-blind, placebo-controlled, two-way crossover study of 26 subjects (Sainati et al 2005; Kryger et al 2008). The oxygen saturation for each hour and for the entire night was comparable between the ramelteon 16 $\mathrm{mg}$ and placebo, suggesting no respiratory depressant effects in these subjects. The mean oxygen saturation values for ramelteon $16 \mathrm{mg}$ and placebo nights during non-REM sleep were 92.9 and 92.7, and during REM sleep the values were 92.5 and 92.1, respectively. A similarly designed study of 26 mild-to-moderate obstructive sleep apnea subjects (apneahypopnea index (AHI) range 5 through 20) given ramelteon $16 \mathrm{mg}$ and placebo on different nights showed no exacerbation of the sleep-disordered breathing (AHI 11.4 and 11.1, respectively) (Kryger et al 2007). Oxygen saturation throughout the night and during specific sleep stages was similar for the placebo and ramelteon $16 \mathrm{mg}$ nights, except for a slight increase during REM sleep for the ramelteon night.

Patients with mild, moderate, and severe renal impairment, including subjects treated with dialysis, were studied following single and multiple doses of ramelteon $16 \mathrm{mg}$ (Tolbert et al 2004d). There was no correlation between the degree of renal impairment and the systemic exposure of ramelteon. Therefore, no ramelteon dosage adjustment is recommended in patients with renal impairment.

The potential for hepatic disease to increase the systemic exposure to ramelteon was demonstrated in a study of patients with mild and moderate hepatic impairment in comparison with healthy matched control subjects following administration of single and multiple doses of ramelteon $16 \mathrm{mg}$ (Karim et al 2004f). Caution is advised in using ramelteon in patients with moderate hepatic impairment, and ramelteon use is not recommended in patients with severe impairment (Takeda Pharmaceuticals North America 2006).

\section{Potential endocrine rffects}

The effects of ramelteon on endocrine function in humans initially was evaluated in a randomized study of 99 healthy subjects given nightly doses of ramelteon $16 \mathrm{mg}$ or placebo for 28 days (Tolbert et al 2004e). Relative to baseline values, there were no significant changes in any of the 12 thyroid, reproductive, and adrenal endocrine parameters measured. 
Potential endocrine effects of longer-term nightly use of ramelteon $16 \mathrm{mg}$ were investigated in a placebo-controlled, 6-month study of 122 chronic insomnia subjects (Richardson et al 2006c). Among the 12 parameters evaluated, there were no significant changes in the thyroid and adrenal axes. Gonadotropins and testosterone were unchanged. Relative to baseline, there was an elevation of the mean serum prolactin level observed only in women. The mean prolactin values remained in the normal reference range and the effect appeared to be transient. There were no apparent associated physical changes. The placebo and ramelteon groups had similar subject-reported menstrual patterns.

As noted above, selected endocrine parameters were monitored during the one-year open-label study of nightly ramelteon in chronic insomnia subjects (Richardson et al 2006a). The mean endocrine values and changes from baseline, as well as the distribution of values around the mean, were contained within the normal range for all parameters. On at least one monthly visit, adults $(16 \mathrm{mg})$ and older adults $(8 \mathrm{mg})$ demonstrated values outside the normal range for total testosterone $(7.3 \%$ and $6.9 \%)$ and free testosterone $(6.0 \%$ and $13.3 \%$ ). There was no placebo comparison to evaluate a potential medication effect.

\section{Abuse liability}

Preclinical ramelteon abuse liability studies with rhesus monkeys demonstrated that it does not produce benzodiazepine agonist-like discriminative stimulus effects in normal or diazepam-dependent animals (France et al 2006), does not cause significant effects on spontaneous or learned behaviors when given daily and then discontinued (France et al 2006), and has no positive reinforcing effects in intravenous selfadministration experiments (Nishida et al 2005). Together these suggest an absence of physical dependence and abuse liability.

The human abuse liability of ramelteon has been investigated in terms of the likelihood of it being misused and also the liability associated with potential acute impairment in psychomotor and cognitive functioning (Griffith and Johnson 2005). Subjects with known sedative drug abuse history participated in a double blind, crossover study of the acute effects (balance, psychomotor, and cognitive tasks) and drug likeability of ramelteon $(16,80,160 \mathrm{mg})$ in comparison with the active control triazolam $(0.25,0.5,0.75 \mathrm{mg})$ (Johnson et al 2006). With ramelteon administration up to 20 times the recommended therapeutic dose there was no differentiation from placebo in any of the behavioral and cognitive performance measures, subjective effect responses, or observer-rated measures. These data confirm the absence of human abuse liability with ramelteon.

\section{Comments}

Ramelteon is the first FDA-approved sleep-promoting medication with a unique mechanism of action that has been released in over 30 years. It is a melatonin receptor agonist, but is different from exogenous melatonin in its structure, receptor subtype selectivity, binding affinity, elimination half-life, metabolism, and efficacy. Moreover, exogenous melatonin is unregulated, while the FDA approval process has required an extensive evaluation of the safety and efficacy of ramelteon.

Ramelteon also is readily differentiated from the BZRA hypnotics, which represent all of the other medications approved for the treatment of insomnia. Ramelteon is unscheduled by the Drug Enforcement Agency (DEA), while the BZRA hypnotics all are schedule IV controlled substances. It has been shown to have no abuse potential, tolerance, rebound insomnia on discontinuation, and no psychomotor or cognitive impairment. Ramelteon promotes sleep through its agonist activity at the $\mathrm{MT}_{1}$ and $\mathrm{MT}_{2}$ melatonin receptor subtypes and thereby attenuates the normal evening SCN arousal signal and reinforces the phase of the circadian system. These actions should shorten sleep latency and increase the likelihood of sleepiness occurring at approximately the same time each night.

The patient experience with ramelteon also may be significantly different from the BZRA hypnotics, which act through a sedating effect. This type of medication-induced sedation clusters with potential adverse effects, including cognitive and psychomotor impairments which are not experienced with ramelteon. With the BZRA hypnotics, patients often are aware of the sedation prior to sleep onset and the maximum benefit in enhancing sleep onset and maintenance typically is achieved with the initial dose. Depending on the elimination half-life and whether a controlled-release formulation is taken, BZRA hypnotics should shorten sleep onset and may improve sleep maintenance. In contrast, ramelteon does not promote sedation. Rather, it shortens sleep onset latency and may help maintain sleep during the early part of the night by attenuating the SCN arousal activity. The ramelteon clinical trials demonstrated both objective and subjective efficacy in shortening sleep onset relative to placebo on the first night the medication was taken. However, patients prescribed ramelteon may notice the absence of the sedating effect and may not report optimum improvement until they have been taking the medication nightly for several days or weeks. There may be 
a clinical analogy with antidepressant medications, although the reason for a subjective sense of improvement may be different. For some patients, the $\mathrm{MT}_{2}$ agonist effect with a shift to an earlier phase and a stabilization of the circadian system may account for this pattern of improvement.

The mechanism of action suggests that ramelteon will be most helpful in enhancing sleep onset when taken in the evening as one's bedtime is approaching. The ramelteon prescribing guidelines suggest that it be taken 30 minutes prior to bedtime. The bedtime sleep-promoting effect presumably is dependent on the attenuation of the SCN-generated arousal that is maximal in the evening. While BZRA hypnotics should have a sedating influence and may facilitate sleep onset at any hour, the pharmacodynamic effects of ramelteon may limit the sleep-promoting efficacy to the evening and early nighttime hours. However, further clinical studies will be necessary to establish the sleep-promoting effects of ramelteon at other times throughout the 24-hour cycle.

The FDA-approved indication for ramelteon is for the treatment of insomnia characterized by difficulty with sleep onset. The mechanism of action explains the timing of the sleep-promoting effect. While there may be an increase in total sleep time, there is not necessarily an improvement in sleep maintenance. As is the case with the two BZRA hypnotic medications approved by the FDA in 2005, eszopiclone and controlled-release zolpidem, the ramelteon indication does not incorporate short-term treatment wording. Accordingly, in appropriate clinical situations, the approved ramelteon prescribing guidelines suggest that it may be used for an extended period.

Several features should contribute to the interest in this new sleep-promoting medication. These include the absence of abuse potential, nonscheduled DEA status, lack of cognitive and psychomotor impairment, minimal potential for drug interactions, and the novel mechanism of action. The safety and efficacy of ramelteon have been demonstrated in both adult and elderly subjects. Limitations include the primary efficacy being sleep onset without an improvement in sleep maintenance.

While the current approved ramelteon indication is for the treatment of insomnia, the pharmacodynamic characteristics raise the question of possible efficacy for circadian rhythm disorders and other clinical conditions where stabilization of the sleep-wake cycle would be especially beneficial. The latter might include bipolar disorder and dementia syndromes. Preliminary studies demonstrating the circadian phase-shifting effects of ramelteon are noted above. Patients with the circadian rhythm disorder delayed sleep phase syndrome (DSPS) have persistent difficulty with sleep onset; however, they also have marked difficulty awakening at a desired time the following morning. While ramelteon would be expected to aid the sleep onset, the circadian phase-response relationship of exogenous melatonin and the preliminary ramelteon evidence suggest that it might have a unique role in the treatment of DSPS. In theory, the agonist activity at the $\mathrm{MT}_{1}$ receptor subtype would directly enhance sleep onset. The effect on the $\mathrm{MT}_{2}$ receptor subtype would promote an advance in the circadian system to allow an earlier sleep onset and an ability to awaken more readily the following morning. Ramelteon also might be beneficial for the free-running non-24-hour rhythm blind individuals who are unable to entrain to the natural 24-hour photoperiod. Other potential applications would include helping individuals with jet lag adjust more rapidly to the new photoperiod of their destination and shift workers readapt to nighttime sleep following a schedule requiring sleep during the daytime.

\section{Summary}

Ramelteon is a selective melatonin receptor agonist approved by the FDA for the treatment of insomnia characterized by difficulty with sleep onset. It is unique among approved sleep-promoting medications due to the mechanism of action targeting the activity in the suprachiasmatic nucleus, as well as an absence of sedating effects and abuse liability.

\section{Disclosures}

Dr Neubauer has served as a consultant to Neurocrine Biosciences, Sanofi-Aventis, and Takeda Pharmaceuticals North America.

\section{References}

Amakye DD, Hibberd M, Stevenson SJ. 2004. A phase I study to investigate the absolute bioavailability of a single oral dose of ramelteon (TAK-375) in healthy male subjects. Sleep, 27(Abstract Suppl):A54.

DeMicco M, Wang-Weigand S, Zhang J. 2006. Long-term therapeutic effects of ramelteon treatment in adults with chronic insomnia: A 1-year study. Sleep, 29(Abstract Suppl):A234.

Erman M, Seiden D, Zammit G, et al. 2006. An efficacy, safety, and doseresponse study of ramelteon in patients with chronic primary insomnia Sleep Med, 7:17-24.

France CP, Weltman RH, Koek W, et al. 2006. Acute and chronic effects of ramelteon in rhesus monkeys (macaca mulatta): dependence liability studies. Behav Neurosci, 120:535-41.

Greenblatt DJ, Harmatz JS, Karim A. 2007. Age and gender effects on the pharmacokinetics and pharmacodynamics of ramelteon, a hypnotic agent acting via melatonin receptors MT1 and MT2.J Clin Pharmacol, 47:485-96.

Griffiths RR, Johnson MW. 2005. Relative abuse liability of hypnotic drugs: A conceptual framework and algorithm for differentiating among compounds. J Clin Psychiatry, 66(Suppl 9):31-41. 
Hajak G, Ebrahim I, Hibberd M, et al. 2007. Ramelteon, unlike zopiclone, has no effect on body sway at peak plasma levels in insomnia patients. Sleep, 30(Abstract Suppl):A245.

Hibberd M, Stevenson SJ. 2004. A phase-I open-label study of the absorption, metabolism, and excretion of (14C)-ramelteon (TAK-375) following a single oral dose in healthy male subjects. Sleep, 27(Abstract Suppl):A54.

Hirai K, Kita M, Ohta H, et al. 2005. Ramelteon (TAK-375) accelerates reentrainment of circadian rhythm after a phase advance of the lightdark cycle in rats. J Biol Rhythms, 20:27-37.

Johnson M, Suess P, Griffiths R. 2006. Ramelteon: a novel hypnotic lacking abuse liability and sedative effects. Arch Gen Psychiatry, 63:1149-57.

Karim A, Bradford D, Siebert F, et al. 2007b. Pharmacokinetic effect of multiple oral doses of donepezil on ramelteon, and vice versa, in healthy adults. Sleep, 30(Abstract Suppl):A244.

Karim A, Bradford D, Zhao Z. 2006d. Effect of multiple oral doses of venlafaxine on the systemic availability of ramelteon, an $\mathrm{MT}_{1} / \mathrm{MT}_{2}$ receptor agonist. Int J Neuropsychopharmacol, 9(Suppl 1):S138.

Karim A, Bradford D, Zhao Z, et al. 2006c. Effect of multiple oral doses of sertraline on the systemic availability of ramelteon, an $\mathrm{MT}_{1} / \mathrm{MT}_{2}$ receptor agonist. J Clin Pharmacol, 46:1090. Abstract 120.

Karim A, Bradford D, Siebert F, et al. 2006b. Effect of multiple doses of escitalopram on the systemic exposure of ramelteon, a selective MT1/MT2 receptor agonist in healthy adults. Sleep, 29(Abstract Suppl):A237.

Karim A, Cao C, Zhao Z, et al. 2004e. Pharmacokinetic interaction between ramelteon (TAK-375) and ethanol in healthy adults. AAPS Pharm Sci, 6. Available from: www.aapspharmsci.org/.

Karim A, Moore R, Zhen Zhao Z, et al. 2007a. Open-label assessment of the pharmacokinetics of ramelteon $8 \mathrm{mg}$ and zolpidem $10 \mathrm{mg}$ coadministration in healthy adults. J Clin Pharmacol, 47:1206. Abstract 103.

Karim A, Tolbert D, Cao C. 2006a. Disposition kinetics and tolerance of escalating single doses of ramelteon, a high-affinity MT1 and MT2 melatonin receptor agonist indicated for treatment of insomnia. J Clin Pharmacol, 46:140-8.

Karim A, Tolbert D, Cao C, et al. 2004a. Effect of food on the systemic exposure of ramelteon (TAK-375) following a single dose. J Clin Pharmacol, 44:1210.

Karim A, Tolbert D, Cao C, et al. 2004b. The effect of multiple doses of ramelteon (TAK-375) on the single-dose pharmacokinetic profile of midazolam in healthy adult subjects. Sleep, 27(Abstract Suppl): A47-A48.

Karim A, Tolbert D, Cao C, et al. 2004c. A multi-dose open-label study to evaluate the effect of rifampin on the pharmacokinetics of ramelteon (TAK-375) in healthy men and women. AASP Pharm Sci, 6. Available from: www.aapspharmsci.org/.

Karim A, Tolbert D, Cao C, et al. 2004d. Effects of fluconazole and ketoconazole on the pharmacokinetics of ramelteon (TAK-375) in normal healthy male and female subjects. Sleep. 27 (Abstract Suppl): A53-A54.

Karim A, Tolbert D, Cao C, et al. 2005a. Study to assess the steady-state drug-drug interaction of omeprazole with ramelteon in healthy adults. Sleep, 28(Abstract Suppl):A46.

Karim A, Tolbert D, Cao C, et al. 2005b. Open-label assessment of the pharmacokinetics and pharmacodynamics of warfarin in the presence of multiple doses of ramelteon in healthy adults. Sleep, 28(Abstract Suppl):A45-A46.

Karim A, Tolbert D, Zhao Z. 2004f. Single and multiple dose pharmacokinetic evaluation of ramelteon (TAK-375) in subjects with and without hepatic impairment. J Clin Pharmacol, 44:1210.

Kato K, Hirai K, Nishiyama K, et al. 2005. Neurochemical properties of ramelteon (TAK-375), a selective MT1/MT2 receptor agonist. Neuropharmacology, 48:301-10.

Kryger M, Wang-Weigand S, Zhang J, et al 2008. Effect of ramelteon, a selective MT1/MT2 receptor-agonist, on respiration during sleep in COPD subjects. Sleep Breath. In press.
Kryger M, Wang-Weigand S, Roth T. 2007. Safety of ramelteon in individuals with mild to moderate obstructive sleep apnea. Sleep Breath, 11:159-64.

Mini L, Wang-Weigand S, Zhang J. 2007. Self-reported efficacy and tolerability of ramelteon $8 \mathrm{mg}$ in older adults experiencing severe sleeponset difficulty. Am J Geriatr Pharmacother, 5:177-84.

Miyamoto M, Nishikawa H, Doken Y, et al. 2004. The sleep-promoting action of ramelteon (TAK-375) in freely moving cats. Sleep, 27:1319-25.

National Institutes of Health. 2005. National institutes of health state of the science conference statement on manifestations and management of chronic insomnia in adults, June 13-15, 2005. Sleep, 28:1049-57.

Nishida N, Sasaki M, Wakasa Y, et al. 2005. Reinforcing effect of ramelteon assessed by intravenous self-administration experiments in rhesus monkeys. Sleep, 28(Abstract Suppl):A45.

Richardson GS. 2005. The human circadian system in normal and disordered sleep. J Clin Psychiatry, 66:3-9.

Richardson G, Zammit G, Rodriquez L, et al. 2005. Evaluation of circadian phase-shifting effects of ramelteon in healthy subjects. Turkey: Society for Chronobiology.

Richardson GS, Wang-Weigand S, Sainati S, et al. 2006c. A double-blind, placebo-controlled Phase III study of the long-term effects of ramelteon on endocrine function in adults with chronic insomnia. Sleep, 29(Abstract Suppl):A232.

Richardson GS, Wang-Weigand S, Zhang J, et al. 2006a. Long-term safety of ramelteon treatment in adults with chronic insomnia: A 1-year study. Sleep, 29(Abstract Suppl):A233.

Richardson G, Zee P, Wang-Weigand S, Rodriguez L, et al. 2006b. Circadian phase-shifting effects of daily ramelteon in healthy adults. Society for Research of Biological Rhythms 10th Biennial Meeting Program and Abstracts. May 21-25:131-2. Abstract 196.

Roth T, Seiden D, Wang-Weigand S, et al. 2007. A 2-night, 3-period, crossover study of ramelteon's efficacy and safety in older adults with chronic insomnia. Curr Med Res Opin, 23:1005-14.

Roth T, Stubbs C, Walsh JK. 2005. Ramelteon (TAK-375), a selective MT1/MT2-receptor agonist, reduces latency to persistent sleep in a model of transient insomnia related to a novel sleep environment. Sleep, 28:303-7.

Roth T, Seiden D, Sainati S, et al. 2006. Effects of ramelteon on patientreported sleep latency in older adults with chronic insomnia. Sleep Med, 7:312-18.

Sainati SM, Karim A, Tolbert D, et al. 2004. Effects of multiple doses of fluoxetine on the systemic exposure of a single dose of ramelteon (TAK375) in healthy adults. Sleep, 27(Abstract Suppl):A48

Sainati S, Tsymbalov S, Demissie S, et al. 2005. Double-blind, placebocontrolled, two-way crossover study of ramelteon in subjects with mild to moderate chronic obstructive pulmonary disease. Sleep, 28(Abstract Suppl):A162.

Takeda Pharmaceuticals North America. 2006. Rozerem prescribing information.

Tolbert D, Karim A, Zhao Z. 2004d. Evaluation of the single and multiple dose pharmacokinetics of ramelteon (TAK-375) in subjects with and without renal impairment. J Clin Pharmacol,.44:1210.

Tolbert D, Karim A, Cao C, et al. 2004a. Study to assess drug interaction between ramelteon (TAK-375) and dextromethorphan in healthy adults. Sleep, 27(Abstract Suppl):A50.

Tolbert D, Karim A, Cao C, et al. 2004b. Multiplle-dose study to assess the effect of ramelteon (TAK-375) on the pharmacokinetics of digoxin in healthy subjects. AAPS Pharm Sci, 6. Available from: www.aapspharmsci.org/.

Tolbert D, Karim A, Johnson J, et al. 2004c. Two-period crossover study to assess the drug interaction between ramelteon (TAK-375) and theophylline in healthy adults. Sleep, 27(Abstract Suppl):A48.

Tolbert D, Karim A, Demissie S. 2004e. A phase I study to evaluate the short-term effects of ramelteon (TAK-375) on endocrine function in healthy adult subjects. $J$ Clin Pharmacol, 44:1012. 
Turek FW, Gillette MU. 2004. Melatonin, sleep, and circadian rhythms: Rationale for development of specific melatonin agonists. Sleep Med, 5:523-32.

Wang-Weigand S, Mayer G, Roth-Schechter B. 2007. Long-term efficacy and safety of ramelteon $8 \mathrm{mg}$ treatment in adults with chronic insomnia: results of a six-month, double-blind, placebo-controlled, polysomnography trial. Sleep Biol Rhythms, 5(Suppl 1):A156. Abstract PO525.

Wang-Weigand S, Zammit G, Peng X. 2007. Plabeco-controlled, doubleblind trial examining the effects of ramelteon vs placebo with zolpidem as a reference on balance in older adults after middle-of-the-night awakenings. Presented at the Annual Meeting of the American Psychiatric Association, San Diego, CA. May, 2007. Abstract NR604.

Yukuhiro N, Kimura H, Nishikawa H, et al. 2004. Effects of ramelteon (TAK-375) on nocturnal sleep in freely moving monkeys. Brain Res, 1027:59-66.

Zammit G, Erman M, Wang-Weigand S, et al. 2007. Evaluation of the efficacy and safety of ramelteon in subjects with chronic insomnia. J Clin Sleep Med, 3:495-504. 
